\title{
Estimación de la prevalencia de riesgo de síndrome de apneas obstructivas del sueño en funcionarios de una Institución de Salud según el cuestionario STOP-Bang
}

\author{
CÉSAR MAQUILÓN O.*, GABRIELA BOFILL B. ${ }^{a}$, PAULINA FUENZALIDA S. ${ }^{a}$, \\ ÁNGELA BENAVENTE O. ${ }^{a}$, MACARENA URRA C. ${ }^{a}$, NICOLÁS VALDÉS O. ${ }^{b}$, ÁNGEL VARGAS D.**, \\ GEORGINA PAVEZ G. ${ }^{\mathrm{c}}$, FELIPE RIVERA CH.* y MÓNICA ANTOLINI T.*
}

Estimation of the prevalence of risk of obstructive sleep apnea syndrome in workers of a health institution according to the stop-bang questionnaire

Introduction: In Chile, it is estimated that $8.5 \%$ of adults are at high risk of Obstructive Sleep Apnea (OSA). Objective: To estimate the risk of OSA in health workers. Material and Method: clinical and anthropometric data, blood pressure, STOP-Bang (CSB) questionnaires, Flemons index and Epworth and Thornton scales were assessed in workers from Clínica Dávila, Santiago, Chile. Results: 1,332 workers were evaluated, $77.1 \%$ women, neck circumference: $35.7 \pm 3.7 \mathrm{~cm}$ (26-54), waist circumference: $89.3 \pm 3.7 \mathrm{~cm}$, and body mass index: $27.5 \pm 4.5$ (17.5-49.4) $\mathrm{kg} / \mathrm{m}^{2} ; 42 \%$ were overweight and $26 \%$ obese. The SB Questionnaire classified them into three risk categories: High risk (HR): 43 workers (3.2\%), $50 \pm 10.5$ years-old; moderate risk: 215 (16.1\%), $45.6 \pm 11.4$ years-old and low risk: 1,074 $(80.6 \%), 36.2 \pm 10.6$ years-old. In men, age and sleep questionnaire scores were different in the three risk categories $(p=0.003$ and 0.001$)$. In women, the sleep questionnaire scores were different in the three risk groups, there were no differences in the Epworth scale $(p=0.274)$, nor in age $(p=0.08)$. The Mallampati scale did not allow predict OSA risk in both sexes. Conclusions: The SB questionnaire identified $9.8 \%$ of the men with high risk of OSA. In men, age, SB questionnaire, Flemons index, Epworth and Thornton scale, were different in the three OSA risk categories. In women, the age and Epworth scale were similar in the different risk categories.

Key words: Sleep apnea, Obstructive; Surveys and questionnaires; Prevalence; Humans; Adults; Body Mass Index, Anthropometry.

\section{Resumen}

Introducción: En Chile, se estima que 8,5\% de los adultos tiene riesgo elevado de síndrome de apnea e hipopnea obstructiva del sueño (SAHOS). Objetivo: Estimar el riesgo de SAHOS en funcionarios de la salud. Material y método: Se consignaron los datos clínicos, antropométricos, presión arterial, cuestionarios STOP-Bang (CSB), indice de Flemons y escalas de Epworth y de Thornton en trabajadores de Clínica Dávila, Santiago, Chile. Resultados: Se evaluaron 1.332 funcionarios, 77,1\% mujeres, circunferencia de cuello: 35,7 $\pm 3,7 \mathrm{~cm}$ (26-54), circunferencia de cintura: 89,3 $\pm 3,7 \mathrm{~cm}$, e índice de masa corporal: $27,5 \pm 4,5(17,5-49,4) \mathrm{kg} / \mathrm{m}^{2} ; 42 \%$ tenía sobrepeso y $26 \%$ obesidad. El Cuestionario $S B$ los clasificó en tres categorías: Riesgo alto (RA): 43 funcionarios (3,2\%), $50 \pm 10,5$ años; riesgo moderado: 215 (16,1\%), 45,6 $\pm 11,4$ años y riesgo bajo: 1.074 (80,6\%), 36, $2 \pm 10,6$ años. En hombres, la edad y los puntajes de los cuestionarios de sueño fueron diferentes en las tres categorías de riesgo $(p=0,003$ y 0,001). En mujeres, los puntajes de los cuestionarios fueron distintos en los tres grupos

\footnotetext{
* Médico, Broncopulmonar de adultos, Servicio de Enfermedades Respiratorias, Clínica Dávila. Santiago, Chile.

** Médico, Internista e Intensivista, jefe del Servicio Médico Quirúrgico, Clínica Dávila. Santiago, Chile. Investigador, Alumno Escuela de Medicina, Universidad de los Andes. Santiago, Chile.

Bioestadístico, Facultad de Enfermería y Obstetricia, Universidad de los Andes. Santiago, Chile.

Enfermera, Coordinadora del Servicio Médico Quirúrgico, Clínica Dávila. Santiago, Chile.
} 
de riesgo, no hubo diferencias en la escala de Epworth $(p=0,274)$, ni en la edad $(p=0,08)$. La escala Mallampati no permitió predecir el riesgo de SAHOS en ambos sexos. Conclusiones: El cuestionario SB identificó a 9,8\% de los hombres con riesgo alto de SAHOS. En los hombres, la edad, cuestionario $S B$, Flemons, Epworth y Thornton, fueron diferentes en las tres categorias de riesgo de SAHOS. En las mujeres, la edad y escala de Epworth fueron similares en las tres categorías de riesgo.

Palabras clave: Apnea obstructiva del sueño; Encuestas y cuestionarios; Prevalencia; Seres humanos; Adultos; Indice masa corporal; Antropometría.

\section{Introducción}

El síndrome de apnea e hipopnea obstructiva del sueño (SAHOS) es uno de los trastornos respiratorios del sueño más frecuente en adultos, con una prevalencia estimada de $4 \%$ en hombres y $2 \%$ en mujeres ${ }^{1}$. Un estudio reciente estima que la prevalencia de este síndrome en grados moderados a severos es $23,4 \%$ en mujeres y $49,7 \%$ en hombres mayores de 40 años de edad ${ }^{2}$. Su síntoma cardinal es la somnolencia diurna excesiva que, junto a la alteración del ánimo y deterioro cognitivo, producen una alteración progresiva en la calidad de vida de los pacientes, y un aumento de la morbilidad cardiovascular ${ }^{3}$. Carrillo et al. usando los datos de la Encuesta Nacional de Salud (ENS) 4 del año 2010 y el cuestionario STOPBang, reveló que a nivel nacional las prevalencias de riesgo medio y alto de SAHOS fueron $32,5 \%$ y $8,5 \%$ respectivamente; el riesgo de SAHOS fue mayor en hombres, aumentando en la medida que la edad y el exceso de peso ascendían, y en forma inversamente proporcional al nivel educacional. Destaca que en nueve regiones de Chile el riesgo alto en hombres supera el 10\% (en siete de ellas supera el $15 \%$ y en dos el $20 \%$ ), siendo los valores de prevalencia de riesgo alto en la Región Metropolitana de $12,2 \%$ en hombres y $4,1 \%$ en mujeres ${ }^{5}$. Entre los factores de riesgo asociados al desarrollo de SAHOS, el sobrepeso y la obesidad son muy importantes, en Chile las últimas tres encuestas de salud realizadas los años, 2003, 2010 y 2017 , muestran un ascenso sostenido en la prevalencia de la obesidad de 21,9\%, 25,1\% y $31,2 \%$ respectivamente.

Los principales factores de riesgo de SAHOS son la edad mayor de 40 años, sexo masculino, índice de masa corporal (IMC) aumentado, diabetes mellitus tipo 2, uso crónico de alcohol y otros $^{6}$; siendo la obesidad el principal factor de riesgo constitucional en SAHOS ${ }^{7}$. En el examen físico, se consideran como datos asociados a mayor probabilidad de padecer SAHOS, la circunferencia cervical y la clasificación de Mallampati del examen de la cavidad oral ${ }^{8}$.

La polisomnografía (PSG) es el 'estándar de oro' para el diagnóstico de este síndrome, pero debido a su alto costo y baja disponibilidad, se han creado protocolos de tamizaje, para detectar a los pacientes con alta probabilidad de padecer esta condición ${ }^{2}$ y así optimizar el uso de los recursos. Los principales cuestionarios disponibles para estimar el riesgo de SAHOS son: STOPBang, Berlín, escala de somnolencia de Epworth (ESE) y Flemons, los cuales consideran variables antropométricas y síntomas, permitiendo determinar los pacientes con riesgo moderado o alto de SAHOS que pueden ser sometidos a estudios simplificados, portátiles y domiciliarios, para confirmar el diagnóstico. Las encuestas más empleadas son aquellas con mayor sensibilidad y especificidad, y con el porcentaje más bajo de falsos positivos, STOP-Bang y Flemons son aquellas que cumplen con estas características.

\section{Objetivos}

Objetivo principal: Estimar el riesgo de SAHOS entre los funcionarios de la Clínica Dávila de Santiago, mediante la aplicación de escalas clínicas predictivas, en una muestra representativa de ellos.

Objetivos secundarios: Estimar según modalidad laboral con turnos nocturnos o sin ellos, el nivel educacional, la prevalencia de ronquido, tabaquismo, sobrepeso, obesidad, hipertensión arterial, saturación de oxígeno y parámetros antropométricos.

\section{Sujetos y Método}

Estudio clínico descriptivo prospectivo observacional que evaluó, la prevalencia de riesgo de SAHOS mediante la aplicación de cuestionarios clínicos en funcionarios adultos mayores de 18 años, de una institución de salud.

Con la autorización del Comité de Ética y de la Gerencia de Recursos Humanos, se invitó a participar a funcionarios de Clínica Dávila; en el año 2017, esta Institución contaba con 642 camas y 3.487 funcionarios, 2.679 eran mujeres 
(76,8\%) y 808 hombres $(23,1 \%)$. Con la hipótesis de una prevalencia de riesgo esperado de SAHOS de $15 \%$, se estableció una muestra a recopilar de 1.330 funcionarios, por ello y asumiendo una pérdida total de encuestas entre 15 y $25 \%$, el tamaño muestral se estimó en alrededor de 1.800 encuestados. La recolección de la información se realizó entre el 15 de octubre y 15 de diciembre de 2017, por nueve estudiantes de medicina de la Universidad de los Andes, quienes estaban familiarizados con este tipo de cuestionarios y encuestas validadas. Toda la información recopilada fue cargada en una planilla Excel, creada para este fin y posteriormente analizada. La duración estimada de las entrevistas realizadas a los funcionarios varió entre 8 y $10 \mathrm{~min}$. Se encuestó un mínimo de 30 funcionarios por cada día de trabajo en la Clínica, durante un período de dos meses.

Se invitó a participar a 2.101 funcionarios, se negaron 381 (tasa de rechazo fue 18,1\%), aceptaron 1.720 funcionarios, hubo 180 encuestas incompletas $(10,4 \%), 1.540$ encuestas fueron válidas para la obtención de los objetivos secundarios, mientras que para los objetivos primarios se consideraron válidas 1.332 encuestas, debido a que 208, tenían al menos una pregunta sin responder en alguno de los cuestionarios auto administrados. Una vez leído y firmado el consentimiento informado y con el participante en posición sentada, se obtuvo la siguiente información: datos sociodemográficos (edad, sexo, nivel educacional, actividad laboral con o sin turnos nocturnos, consumo de tabaco), mediciones antropométricas (índice de masa corporal, circunferencia cervical y de cintura), presión arterial en reposo, escala de Mallampati y los cuestionarios de sueño: Stop-Bang, índice de Flemons, escala de Thornton y escala de somnolencia de Epworth.

Se usaron monitores de constantes vitales marca MINDRAY modelo VS-900 (Shenzhen Mindray Bio-Medical Electronics Co., LTD. Mindray Building, Keji 12th Road South, Hi-Tech Industrial Park, Nanshan, Shenzhen, 518057, P.R. China.). Pesas y tallímetros digitales marca SECA (SECA Deutschland, Hammer Steindamm 3-25, 22089 Hamburgo, Alemania) y cintas inelásticas estándar para medición de perímetros de cuello y abdomen.

A los sujetos con riesgo elevado de SAHOS se les informó acerca de su condición de salud a través del investigador principal.

\section{Análisis estadístico}

Los resultados fueron expresados como valores promedio \pm desviación estándar para las variables numéricas de distribución normal y en porcentaje para las medidas en escala nominal. Las variables cuantitativas fueron resumidas mediante estadígrafos de tendencia central y de dispersión; las variables cualitativas fueron analizadas mediante frecuencias absolutas y relativas. Se evaluó el riesgo relativo mediante regresiones logísticas ordinales, según las variables predictoras.

\section{Resultados}

Las características sociodemográficas y la modalidad laboral de los 1.540 funcionarios se describen en la Tabla 1. La frecuencia de fumadores activos es similar en ambos grupos, al igual que su IMC. Las cifras de presión arterial no muestran diferencias entre los grupos. La frecuencia de roncadores es mayor entre los que realizan sólo actividades diurnas $(\mathrm{p}=0,001)$, mientras que los que realizan más actividad física son aquellos funcionarios que cumplen turnos nocturnos $(p=0,001)$.

Las encuestas válidas para el análisis de riesgo de SAHOS fueron 1.332, (Tabla 2), $1.027 \mathrm{mu}-$ jeres $(77,1 \%)$ y 305 hombres $(22,9 \%)$, de todos los estamentos. Del grupo total, $34,8 \%$ tenían educación universitaria completa, 465 eran fumadores activos $(34,9 \%)$ y $526(39,7 \%)$ funcionarios referían ser roncadores; sólo $449(33,8 \%)$ realizaban actividad física regularmente. El 9,6\% de los funcionarios entrevistados eran hipertensos (ENS 27,6\%) (4). La talla promedio de la muestra fue $1,61 \pm 0,08 \mathrm{~m}$ (rango: 1,42-1,92), y los promedios de peso e IMC fueron $71,5 \pm 13,6 \mathrm{~kg} \mathrm{y}$ $27,5 \pm 4,5 \mathrm{~kg} / \mathrm{m}^{2}$ respectivamente. El grupo con sobrepeso representa el 42,1\% (ENS 39,8\%) y el porcentaje de obesos es 25,9\% (ENS 34,4\%), hay 14 funcionarios con obesidad mórbida o grado III (IMC > 40) representando el 1,1\% de la muestra (ENS 3,2\% $)^{4}$. La circunferencia cervical media fue $35,7 \pm 3,8 \mathrm{~cm}$ (rango de 26 a $54 \mathrm{~cm}$ ) y la circunferencia de cintura promedio fue $89,4 \pm 12,3$ cm (Tabla 2).

Según el cuestionario STOP-Bang (Tabla 3), 43 funcionarios ( $3,2 \%$ de la muestra) tenían riesgo alto de SAHOS, de ellos, 30 eran hombres, los cuales representan al 9,8\% de la muestra total de varones (305 hombres), el valor promedio de STOP-Bang: 5,42 $\pm 0,66$, edad promedio: $48,1 \pm 11,3$ (rango: 27-66). Entre las mujeres encuestadas $(\mathrm{n}=1.027), 13$ de ellas $(1,26 \%)$ tenían un puntaje de STOP-Bang entre 5 y 8 , por tanto, con riesgo elevado de SAHOS, su edad era de 54,6 \pm 6,3 años. Además, 215 funcionarios tenían riesgo medio de SAHOS $(16,1 \%)$, edad 
Tabla 1. Características generales, sociodemográficas y modalidad laboral de $\mathbf{1 . 5 4 0}$ funcionarios encuestados de la Clínica Dávila

\begin{tabular}{|c|c|c|c|}
\hline Descripción de grupo & $\begin{array}{l}\text { Con turnos nocturnos } \\
\qquad(\mathrm{n}=\mathbf{8 5 9})\end{array}$ & $\begin{array}{l}\text { Sin turnos nocturnos } \\
\qquad(\mathrm{n}=681)\end{array}$ & $\begin{array}{c}\text { Total } \\
(\mathrm{n}=\mathbf{1 . 5 4 0})\end{array}$ \\
\hline Masculino & 171 & 190 & 361 \\
\hline Femenino & 688 & 491 & 1.179 \\
\hline Nunca ha fumado & 342 & 301 & 643 \\
\hline Exfumador & 146 & 97 & 243 \\
\hline Fumador activo & 264 & 222 & 486 \\
\hline Presión arterial sistólica (mmHg) & 126,35 & 125,16 & 125,8 \\
\hline Presión arterial diastólica (mmHg) & 77,32 & 77,11 & 77,22 \\
\hline No Roncador & $474(55,2 \%)$ & $422(62 \%)$ & 896 \\
\hline Roncador & $367(42,7 \%)$ & $243(35,7 \%)$ & 610 \\
\hline Saturación de $\mathrm{O}_{2}(\%)$ & 97,8 & 98,2 & 98,0 \\
\hline $\mathrm{IMC}\left(\mathrm{kg} / \mathrm{m}^{2}\right)$ & 27,49 & 27,66 & 27,57 \\
\hline No realiza actividad física & 609 & 421 & 1.030 \\
\hline Si realiza actividad física & 250 & 260 & 510 \\
\hline \multirow[t]{4}{*}{ Nivel educacional } & $185(61 \%)$ & $118(39 \%)$ & 303 \\
\hline & $366(59,3 \%)$ & $251(40,6 \%)$ & 617 \\
\hline & $230(48,2 \%)$ & $247(51,8 \%)$ & 477 \\
\hline & 781 & 616 & 1.397 \\
\hline Encuestados según cargos $(n=1.186)$ & Frecuencia & Porcentaje & Acumulado \\
\hline Técnico de Enfermería (TENS) & 332 & 27,9 & 27,9 \\
\hline Auxiliares de servicio & 213 & 17,9 & 45,9 \\
\hline Administrativo & 188 & 15,8 & 61,8 \\
\hline Enfermeras/Matronas & 166 & 14 & 75,8 \\
\hline Médicos & 145 & 12,2 & 88 \\
\hline Klgos, Tecnólogos, Nutricionistas & 77 & 6,5 & 94,5 \\
\hline Otros Profesionales administrativos & 62 & 5,23 & 99,7 \\
\hline Gerentes & 3 & 0,3 & 100 \\
\hline Total & 1.186 & 100 & \\
\hline
\end{tabular}

*Técnico $=4$ semestres después de enseñanza media. **Superior $=5$ años o más de educación universitaria.

promedio: $46,6 \pm 11,5$, score promedio: $3,30 \pm$ 0,46 y 1.074 funcionarios $(80,6 \%)$ tenían bajo riesgo de SAHOS, edad promedio: $36,3 \pm 10,6$ años y puntaje promedio: $0,86 \pm 0,75$.

El Score Clínico de Apnea del Sueño de Flemons (SCAS) mayor de 15 puntos identificó con Riesgo Alto para SAHOS a 52 funcionarios, 29 hombres, edad 47,1 \pm 10 años (SCAS: $28,1 \pm 15,1$; rango 18-80) y 23 mujeres, edad 47,0 $\pm 10,3$ (SCAS: 20,2 $\pm 3,8$; rango 16-30).

En los hombres los puntajes de los cuestionarios de Flemons, Thornton, Epworth y STOP-Bang difirieron significativamente en los tres grupos de riesgo $(\mathrm{p}<0,001 ; 0,001 ; 0,001 \mathrm{y}$
0,026 respectivamente), en ellos, la edad también fue diferente en las tres categorías de riesgo de SAHOS $(p=0,003)$. Las mujeres tuvieron diferentes puntajes en los cuestionarios de Flemons, Thornton y STOP-Bang en los tres grupos de riesgo para SAHOS $(p<0,001)$; mientras que la edad y los puntajes de la escala de Epworth no fueron diferentes en las tres categorías de riesgo de SAHOS ( $p=0,274$ y 0,08 respectivamente). El puntaje de la evaluación de la escala de Mallampati no mostró diferencias entre los grupos de riesgo en ambos sexos (Tabla 3 ).

La Tabla 4, muestra que el rango de edad entre 30 a 54 años se asocia a mayor riesgo de 
Tabla 2. Características de 1.332 funcionarios de Clínica Dávila que completaron las encuestas de sueño

\begin{tabular}{|c|c|c|}
\hline Variable & & Promedio \pm DE (rango) \\
\hline Edad (años) & & $38,22 \pm 11,49(18-71)$ \\
\hline Sexo & $\begin{array}{l}\text { Masculino } \\
\text { Femenino }\end{array}$ & $\begin{array}{r}305(22,9 \%) \\
1.027(77,1 \%)\end{array}$ \\
\hline Hábito tabáquico $(\mathrm{n}=1.331)$ & $\begin{array}{l}\text { Nunca ha fumado } \\
\text { Exfumador } \\
\text { Fumador activo }\end{array}$ & $\begin{array}{l}628(47,2 \%) \\
238(17,9 \%) \\
465(34,9 \%)\end{array}$ \\
\hline Roncador $(\mathrm{n}=1.326)$ & $\begin{array}{l}\text { No ronca } \\
\text { Si ronca }\end{array}$ & $\begin{array}{l}800(60,3 \%) \\
526(39,7 \%)\end{array}$ \\
\hline Actividad física $(\mathrm{n}=1.329)$ & $\begin{array}{l}\text { No realiza actividad física } \\
\text { Si realiza actividad física }\end{array}$ & $\begin{array}{l}880(66,2 \%) \\
449(33,8 \%)\end{array}$ \\
\hline Nivel educacional $(\mathrm{n}=1.187)$ & $\begin{array}{l}\text { Media } \\
\text { Técnico } \\
\text { Universitario }\end{array}$ & $\begin{array}{l}254(21,4 \%) \\
520(43,8 \%) \\
413(34,8 \%)\end{array}$ \\
\hline Hipertensión arterial $(\mathrm{n}=1.330)$ & $\begin{array}{l}\text { No } \\
\text { Sí }\end{array}$ & $\begin{array}{r}1.203(90,5 \%) \\
127(9,5 \%)\end{array}$ \\
\hline $\begin{array}{l}\text { Datos antropométricos }(\mathbf{n}=\mathbf{1 . 3 3 2}) \\
\text { Índice de Masa Corporal }\left(\mathrm{kg} / \mathrm{m}^{2}\right)\end{array}$ & & $27,53 \pm 4,53(17,51-49,38)$ \\
\hline Categoría IMC $\left[\mathrm{kg} / \mathrm{m}^{2}\right]$ & $\begin{array}{l}<18,5 \text { - Desnutrido } \\
18,5-24,9 \text { - Normal } \\
25-29,9 \text { - Sobrepeso } \\
30-34,9 \text { - Obesidad tipo I } \\
35-39,9 \text { - Obesidad tipo II } \\
>40 \text { - Obesidad tipo III }\end{array}$ & $\begin{array}{rr}3 & (0,23 \%) \\
422 & (31,68 \%) \\
561 & (42,12 \%) \\
259 & (19,44 \%) \\
73 & (5,48 \%) \\
14 & (1,05 \%)\end{array}$ \\
\hline Talla $[\mathrm{m}]$ & & $1,61 \pm 0,08(1,42-1,92)$ \\
\hline Peso $[\mathrm{kg}]$ & & $71,46 \pm 13,60(41-142,7)$ \\
\hline Circunferencia cervical $[\mathrm{cm}]$ & & $35,68 \pm 3,76(26-54)$ \\
\hline Circunferencia de cintura $[\mathrm{cm}]$ & & $89,37 \pm 12,26(55-137)$ \\
\hline
\end{tabular}

SAHOS (Stop Bang: 5 a 8 puntos) (OR: 6,10 IC 95\%: 2,56 a 14,56) y el rango de edad de 55 años o más, se asocia a un riesgo mayor (STOP Bang alto) (OR: 13,51 IC $95 \%: 5,45$ a 33,46$)$. El IMC entre 30 y 34,9 tiene un riego elevado de SAHOS (STOP Bang elevado: 4 a 8 puntos) y este es mayor cuando el IMC se encuentra entre 35 y 39,9 o es mayor de 40 . El perímetro cervical $>41 \mathrm{o}>43 \mathrm{~cm}$ está asociado a STOP Bang alto (OR: 25,48, IC: 13,23 a 49,06). Una encuesta de Flemons mayor de 14 puntos se asocia a riesgo alto de SAHOS (STOP Bang: 5 a 8 puntos) (OR:43,97, IC: 21,85 a 88,45). La clasificación de Mallampati de 3 o 4 no se asocia a un puntaje STOP-Bang elevado (OR: 1,15, IC: 0,62 a 2,14). Se confeccionaron curvas de riesgo según IMC y edad, considerando el STOP-Bang $>4$ (Figuras 1 y 2), mediante un modelo de regresión logística, que establece una relación entre el riesgo de SAHOS (STOP-Bang > 4) y las variables predictoras edad e IMC, este modelo estima la probabilidad de riesgo.

La Tabla 1 describe además a 1.186 funcionarios estratificados según profesión y rol que desempeñan en la Clínica, 388 son profesionales de la salud (enfermeras, matronas, médicos, kinesiólogos, tecnólogos médicos y nutricionistas), 332 son técnicos de enfermería nivel superior (TENS), 65 son otros profesionales administrativos y gerentes, y 401 son administrativos o auxiliares de servicio. A este grupo de 1.186 funcionarios, se les aplicó las encuestas STOP-Bang y Flemons, modificando el puntaje de los 3 grupos de riesgo de SAHOS mediante STOP-Bang: (0 a 1) bajo riesgo; (2 a 3 ) riesgo moderado y (4 a 8) riesgo alto. Se identificaron 35 funcionarios $(2,95 \%)$ de 1.186 con elevado riesgo de SAHOS y la encuesta de Flemons identificó como riesgo alto de SAHOS a 45 funcionarios que representan $3,79 \%$ de esta misma muestra. 
Tabla 3. Estimación del riesgo de SAHOS mediante el cuestionario STOP-Bang según sexo y su relación con otras encuestas de sueño aplicadas a funcionarios de la Clínica Dávila

\begin{tabular}{|c|c|c|c|c|c|c|c|}
\hline \multicolumn{8}{|c|}{ Sexo masculino $($ total $=305)$} \\
\hline \multirow{2}{*}{$\begin{array}{l}\text { Riesgo SAHOS } \\
\text { Estadígrafo }\end{array}$} & \multicolumn{2}{|c|}{$\begin{array}{c}\text { Bajo }(n=182) \\
\text { (STOP-Bang 0-2) }\end{array}$} & \multicolumn{2}{|c|}{$\begin{array}{c}\text { Medio }(n=93) \\
\text { (STOP-Bang 3-4) }\end{array}$} & \multicolumn{2}{|c|}{$\begin{array}{c}\text { Medio }(n=30) \\
\text { (STOP-Bang 5-8) }\end{array}$} & \multirow[t]{2}{*}{$\mathbf{p}$} \\
\hline & Promedio $\pm \mathrm{DE}$ & Rango & Promedio $\pm \mathrm{DE}$ & Rango & Promedio $\pm \mathrm{DE}$ & Rango & \\
\hline Edad (años) & $34,22 \pm 9,18$ & $18-63$ & $43,37 \pm 12,30$ & $20-71$ & $48,13 \pm 11,39$ & $27-66$ & 0,003 \\
\hline STOP-Bang & $1,6 \pm 0,50$ & $0-2$ & $3,30 \pm 0,46$ & $3-4$ & $5,4 \pm 0,67$ & $5-8$ & 0,026 \\
\hline Epworth & $6,06 \pm 3,79$ & $0-18$ & $7,59 \pm 4,46$ & $0-20$ & $10,8 \pm 6,32$ & $2-22$ & 0,001 \\
\hline Thornton & $0,98 \pm 1,63$ & $0-9$ & $3,87 \pm 3,62$ & $0-15$ & $5,50 \pm 3,44$ & $0-12$ & 0,001 \\
\hline Flemons & $3,32 \pm 2,62$ & $0-18$ & $9,23 \pm 6,16$ & $1-38$ & $22,8 \pm 16,89$ & $3-80$ & 0,001 \\
\hline Mallampati & $2,20 \pm 1,04$ & $1-4$ & $2,55 \pm 1,1$ & $1-4$ & $2,43 \pm 1,17$ & $1-4$ & 0,647 \\
\hline$\%$ Muestral & $59,67 \%$ & & $30,49 \%$ & & $9,83 \%$ & & \\
\hline \multicolumn{8}{|c|}{ Sexo femenino $($ total $=1.027)$} \\
\hline Riesgo SAHOS & \multicolumn{2}{|c|}{$\begin{array}{c}\text { Bajo }(n=892) \\
(\text { STOP- Bang 0-2) }\end{array}$} & \multicolumn{2}{|c|}{$\begin{array}{c}\text { Medio (n=122) } \\
\text { (STOP- Bang 3-4) }\end{array}$} & \multicolumn{2}{|c|}{$\begin{array}{c}\text { ALTO (n=13) } \\
(\text { STOP- Bang 5-8) }\end{array}$} & $\mathbf{p}$ \\
\hline Estadígrafo & Promedio $\pm \mathrm{DE}$ & Rango & Promedio $\pm \mathrm{DE}$ & Rango & Promedio $\pm \mathrm{DE}$ & Rango & \\
\hline Edad (años) & $36,69 \pm 10,85$ & $18-69$ & $47,30 \pm 10,51$ & $24-70$ & $54,62 \pm 6,33$ & $36-62$ & 0,08 \\
\hline STOP-Bang & $0,76 \pm 0,76$ & $0-2$ & $3,30 \pm 0,46$ & $3-4$ & $5,46 \pm 0,66$ & $5-7$ & 0,001 \\
\hline Epworth & $8,48 \pm 4,8$ & $0-24$ & $9,45 \pm 5,26$ & $0-21$ & $11,85 \pm 5,74$ & $3-24$ & 0,274 \\
\hline Thornton & $0,89 \pm 1.87$ & $0-15$ & $3,75 \pm 3,58$ & $0-15$ & $5,85 \pm 4,1$ & $0-12$ & 0,001 \\
\hline Flemons & $1,41 \pm 2,15$ & $0-20$ & $6,02 \pm 5,32$ & $0-30$ & $13,46 \pm 7,32$ & $4-25$ & 0,001 \\
\hline Mallampati & $2,19 \pm 1,04$ & $1-4$ & $2,63 \pm 1,04$ & $1-4$ & $2,55 \pm 0,93$ & $1-4$ & 0,892 \\
\hline$\%$ Muestral & $86,85 \%$ & & $11,87 \%$ & & $1,26 \%$ & & \\
\hline
\end{tabular}

Tabla 4. Análisis de las variables asociadas al riesgo elevado de SAHOS (STOP-Bang entre 5 y 8 puntos) ( $n=1.332$ funcionarios)

\begin{tabular}{|c|c|c|c|c|}
\hline STOP-Bang (5 a 8) & Odds ratio & $\mathbf{P}>\mathbf{z}$ & IC95\% & \\
\hline $\begin{array}{l}\text { Edad } \\
\quad 18 \text { a } 29 \text { años (referencia) }\end{array}$ & $\begin{array}{l}1,09 \\
1,00\end{array}$ & 0,00 & 1,06 & 1,12 \\
\hline 30 a 54 años & 6,10 & 0,00 & 2,56 & 14,56 \\
\hline 55 o más años & 13,51 & 0,00 & 5,45 & 33,46 \\
\hline $\begin{array}{l}\text { IMC } \\
\qquad 18,6 \text { a } 24,9 \mathrm{~kg} / \mathrm{m}^{2} \text { (referencia) }\end{array}$ & $\begin{array}{l}1,26 \\
1,00\end{array}$ & 0,00 & 1,19 & 1,34 \\
\hline $25-29,9$ & 3,04 & 0,16 & 0,64 & 14,38 \\
\hline $30-34,9$ & 17,57 & 0,00 & 4,07 & 75,84 \\
\hline $35-39,9$ & 22,27 & 0,00 & 4,53 & 109,52 \\
\hline$>40$ & 157,50 & 0,00 & 27,47 & 903,19 \\
\hline Perímetro cuello (cm) & 1,59 & 0,00 & 1,44 & 1,77 \\
\hline Perímetro cuello $>41 *$ o $>43 * *$ & 25,48 & 0,00 & 13,23 & 49,06 \\
\hline Flemons & 1,28 & 0,00 & 1,22 & 1,34 \\
\hline Flemons $>14$ & 43,97 & 0,00 & 21,85 & 88,45 \\
\hline Thornton & 1,37 & 0,00 & 1,27 & 1,47 \\
\hline Thornton $>4,9$ & 9,22 & 0,00 & 4,94 & 17,20 \\
\hline Mallampati & 1,20 & 0,22 & 0,90 & 1,60 \\
\hline Mallampati $>2$ & 1,15 & 0,65 & 0,62 & 2,14 \\
\hline
\end{tabular}

*Perímetro de cuello asociado a mayor riesgo de SAHOS en mujeres.**Perímetro de cuello asociado a mayor riesgo de SAHOS en hombres'. 

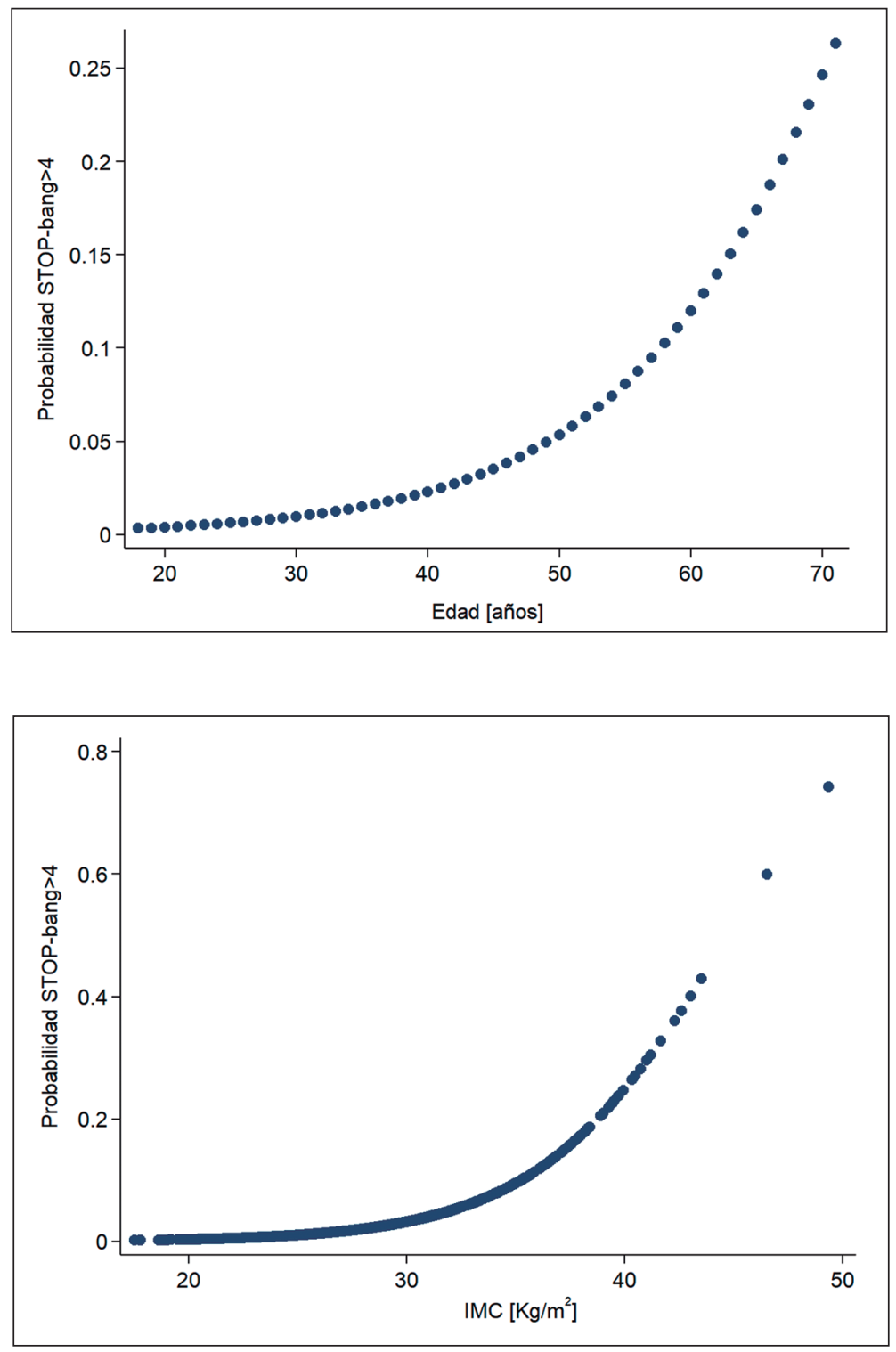

Figura 1. Modelo de regresión logística entre la variable predictora edad y probabilidad de STOP-Bang $>4$ $(\mathrm{n}=1.332$ funcionarios $)$.
Figura 2. Modelo de regresión logística entre la variable predictora índice de masa corporal (IMC) y probabilidad de STOP-Bang $>4(\mathrm{n}=1.332$ funcionarios).
El puntaje de Flemons $<15$, en esta misma muestra (1.1.86 funcionarios) identifica como bajo riesgo de SAHOS a un $96,2 \%$ de la muestra y el STOP-Bang de 0 a 3 (riesgo bajo + riesgo moderado) identifica a un $97 \%$ de los mismos individuos (Tabla 5).

\section{Discusión}

Según los resultados de Carrillo y cols. ${ }^{5}$, el $8,2 \%$ de la población adulta chilena tiene un riesgo elevado de SAHOS, destacando que los hombres de la Región Metropolitana tienen un riesgo alto de SAHOS de 12,2\% (IC 8,8 -16,7), en mujeres el riesgo reportado en Chile es $3,6 \%$.
Nuestra muestra la constituyen principalmente mujeres $(77,1 \%)$ y la edad promedio fue 38,2 $\pm 11,5$ años, inferior a la muestra de la ENS de 2009 que fue $48 \pm 18$ años.

El cuestionario de STOP-Bang tiene elevada sensibilidad para la pesquisa de pacientes con SAHOS, la sensibilidad reportada según el índice de apnea-hipopnea (IA/H) $>5,>15$ y $>30$ ha sido $83,6 \%, 93 \%$ y $100 \%$, respectivamente ${ }^{12}$, $\mathrm{y}$ aunque tiene baja especificidad: $43 \%$ para un IAH de 15 a 30 y $37 \%$ para un IAH $>30^{11}$, no obstante, esta debilidad; el cuestionario de STOP-Bang tiene una de las tasas más bajas de falsos negativos (16\%) entre los cuestionarios de tamizaje para $\mathrm{SAHOS}^{12,13}$.

Saldías y cols., publicaron en el año 2018, un 
Tabla 5. Encuestas de STOP-Bang y Flemons para determinar el riesgo de SAHOS en los funcionarios** según cargo que desempeñan $(n=1.186)$

\begin{tabular}{|c|c|c|c|c|c|c|c|}
\hline Cargo & $\begin{array}{l}\text { STOP-B } \\
\left(\begin{array}{l}0 \\
\text { a }\end{array}\right)\end{array}$ & $\begin{array}{l}g^{*} \\
(2 \text { a } 3)\end{array}$ & (4 a 8$)$ & Total & $\begin{array}{c}\text { Flemons } \\
(<15)\end{array}$ & $(\geq 15)$ & Total \\
\hline Administrativo & $\begin{array}{c}133 \\
70,74\end{array}$ & $\begin{array}{c}45 \\
23,94\end{array}$ & $\begin{array}{c}10 \\
5,32\end{array}$ & $\begin{array}{l}188 \\
100\end{array}$ & $\begin{array}{c}174 \\
92,55\end{array}$ & $\begin{array}{c}14 \\
7,45\end{array}$ & $\begin{array}{l}188 \\
100\end{array}$ \\
\hline Técnico de enfermería nivel superior & $\begin{array}{c}260 \\
78,31\end{array}$ & $\begin{array}{c}61 \\
18,37\end{array}$ & $\begin{array}{c}11 \\
3,31\end{array}$ & $\begin{array}{l}332 \\
100\end{array}$ & $\begin{array}{c}319 \\
96,08\end{array}$ & $\begin{array}{c}13 \\
3,92\end{array}$ & $\begin{array}{l}332 \\
100\end{array}$ \\
\hline Auxiliares de servicio & $\begin{array}{c}182 \\
85,45\end{array}$ & $\begin{array}{c}30 \\
14,08\end{array}$ & $\begin{array}{c}1 \\
0,47\end{array}$ & $\begin{array}{l}213 \\
100\end{array}$ & $\begin{array}{c}209 \\
98,12\end{array}$ & $\begin{array}{c}4 \\
1,88\end{array}$ & $\begin{array}{l}213 \\
100\end{array}$ \\
\hline Enfermeras/matronas & $\begin{array}{c}144 \\
86,75\end{array}$ & $\begin{array}{c}18 \\
10,84\end{array}$ & $\begin{array}{c}4 \\
2,41\end{array}$ & $\begin{array}{l}166 \\
100\end{array}$ & $\begin{array}{c}161 \\
96,99\end{array}$ & $\begin{array}{c}5 \\
3,01\end{array}$ & $\begin{array}{l}166 \\
100\end{array}$ \\
\hline Gerentes & $\begin{array}{c}3 \\
100\end{array}$ & $\begin{array}{l}0 \\
0\end{array}$ & $\begin{array}{l}0 \\
0\end{array}$ & $\begin{array}{c}3 \\
100\end{array}$ & $\begin{array}{c}3 \\
100\end{array}$ & $\begin{array}{l}0 \\
0\end{array}$ & $\begin{array}{c}3 \\
100\end{array}$ \\
\hline Médicos & $\begin{array}{l}126 \\
86,9\end{array}$ & $\begin{array}{c}15 \\
10,34\end{array}$ & $\begin{array}{c}4 \\
2,76\end{array}$ & $\begin{array}{l}145 \\
100\end{array}$ & $\begin{array}{c}141 \\
97,24\end{array}$ & $\begin{array}{c}4 \\
2,76\end{array}$ & $\begin{array}{l}145 \\
100\end{array}$ \\
\hline Otros profesionales administrativos & $\begin{array}{c}57 \\
91,94\end{array}$ & $\begin{array}{c}3 \\
4,84\end{array}$ & $\begin{array}{c}2 \\
3,23\end{array}$ & $\begin{array}{c}62 \\
100\end{array}$ & $\begin{array}{c}60 \\
96,77\end{array}$ & $\begin{array}{c}2 \\
3,23\end{array}$ & $\begin{array}{c}62 \\
100\end{array}$ \\
\hline Otros profesionales de salud & $\begin{array}{c}52 \\
67,53\end{array}$ & $\begin{array}{c}22 \\
28,57\end{array}$ & $\begin{array}{c}3 \\
3,9\end{array}$ & $\begin{array}{c}77 \\
100\end{array}$ & $\begin{array}{c}74 \\
96,1\end{array}$ & $\begin{array}{c}3 \\
3,9\end{array}$ & $\begin{array}{c}77 \\
100\end{array}$ \\
\hline Total & $\begin{array}{c}957 \\
80,69\end{array}$ & $\begin{array}{c}194 \\
16,36\end{array}$ & $\begin{array}{c}35 \\
2,95\end{array}$ & $\begin{array}{c}1186 \\
100\end{array}$ & $\begin{array}{c}1141 \\
96,21\end{array}$ & $\begin{array}{c}45 \\
3,79\end{array}$ & $\begin{array}{c}1186 \\
100\end{array}$ \\
\hline
\end{tabular}

*El rango de STOP-Bang se modificó a categorías (0 a 1) (2 a 3) y (4 a 8), el índice de Flemons no se modificó. **Se excluyeron 146 funcionarios, correspondiente al 10,96\% del total de la muestra $(\mathrm{n}=1.332$ funcionarios) con datos incompletos, no fue posible agruparlos en los cargos descritos en la tabla.

análisis de 1.050 roncadores chilenos de ambos sexos, les aplicaron todas las encuestas validadas para la detección de SAHOS, luego una poligrafía domiciliaria y con el diagnóstico de SAHOS analizaron la sensibilidad y especificidad de las encuestas, así como los valores predictivos y concluyen que el perímetro cervical fue el principal hallazgo clínico asociado al riesgo de SAHOS (AUC: 0,70; IC95\%: 0,67 a 0,73; p < 0,0001), además describen que la sensibilidad del Índice de Flemons fue 0,40, la especificidad de 0,82 y el VPP de 0,92. Mientras que la encuesta STOPBang tiene una sensibilidad de 0,95 , una especificidad de 0,21 y un VPP de 0,86 ${ }^{14}$.

En nuestro estudio al clasificar a los funcionarios según el cuestionario de STOP-Bang, encontramos que un $9,8 \%$ de los varones (30 funcionarios) y 13 mujeres tenían riesgo elevado de SAHOS. Mientras que el índice de Flemons de 15 o más puntos detectó SAHOS severo en 52 funcionarios, 29 hombres y 23 mujeres. Los cuatro cuestionarios de sueño (STOP-Bang, Epworth, Thornton y Flemons) tuvieron diferentes puntajes en las tres categorías de riesgo de SAHOS en los varones; lo mismo aconteció en las mujeres excepto para la escala de Epworth. En un estu- dio realizado en 3.093 pacientes colombianos examinados entre el 2005 y 2009 , no hubo correlación entre el puntaje de la escala de Epworth y el índice de apnea e hipopnea, tampoco hubo correlación al estratificarlo por edad y sexo ${ }^{15}$. La escala de somnolencia de Epworth fue diseñada por Johns y cols. en 1991, el estudio incluyó a 180 sujetos, 30 controles y 150 pacientes con diferentes trastornos del sueño (SAHOS, narcolepsia e hipersomnia idiopática), en nuestro grupo de funcionarios analizados no hubo diferencias significativas en las tres categorías de riesgo para SAHOS en mujeres.

Debemos considerar las limitaciones de nuestro estudio, no es un estudio aleatorio, el temor de algunos funcionarios a entregar información sobre las características de su sueño al dudar de la confidencialidad de la información, puede ser un sesgo; además no contamos con un estudio diagnóstico validado, realizado en forma simultánea para cotejar los resultados de las encuestas; sin embargo, el estudio de Saldías y cols. ${ }^{14}$ realizado en población chilena describe la relevancia y valor de estas encuestas.

Por otro lado, la escala de Mallampati no mostró diferencias significativas entre los grupos 
de riesgo de SAHOS en ambos sexos. La escala de Mallampati es un sistema de clasificación simple de la vía aérea empleado en los servicios de anestesiología, para identificar a los sujetos adultos que tienen mayor riesgo de intubación traqueal difícil, es un sistema simple, no invasivo y fácil de aprender. Sin embargo, su utilidad en la pesquisa de pacientes con SAHOS ha sido motivo de controversia, y aunque en nuestro análisis no encontramos diferencias entre las tres categorías de riesgo en hombres y mujeres, hay estudios en otros grupos de individuos en que si fue útil como predictor $^{16}$.

\section{Conclusiones}

El empleo de los cuestionarios de sueño y la medición de datos antropométricos son una herramienta objetiva y reproducible para que médicos generales y de familia puedan detectar a las personas con mayor riesgo de prevalencia de SAHOS, especialmente los más severos y dirigir los esfuerzos diagnósticos hacia ese grupo. Considerando la relevancia del perímetro de cuello como determinante del examen físico para riesgo de SAHOS, esta medición debería mantenerse en la encuesta nacional de salud, ya que se excluyó de la última encuesta nacional del año 2017.

\section{Bibliografía}

1.- Uso de CPAP nasal en el largo plazo en síndrome de apnea-hipopnea del sueño [Internet]. [citado 3 de agosto de 2018]. Disponible en: https:// scielo.conicyt.cl/scielo.php?script=sci_arttext\&pid $=$ S0034-98872007000700005.

2.- GARBARINO S, GUGLIELMI O, SANNA A, MANCARDI GL, MAGNAVITA N. Risk of occupational accidents in workers with obstructive sleep apnea: systematic review and meta-analysis. Sleep 2016; 39: 1211-8.

3.- LLOBERS P, DURÁN-CANTOLLA J, MARTÍNEZGARCÍA MA, MARIN JM, FERRER A, et al. Diagnosis and treatment of sleep apnea-hypoapnea syndrome. Spanish Society of Pulmonology and Thoracic Surgery. Arch Bronconeumol 2011; 47: 143-56.

4.- ENS - Encuesta Nacional de Salud - EPI - Departamento de Epidemiologia [Internet]. [citado 4 de agosto de 2018]. Disponible en: http://epi.minsal.cl/encuesta-ensanteriores/

5.- CARRILLO AJ, VARGAS RC, CISTERNAS VA, OLIVARES-TIRADO P. Prevalencia de riesgo de apnea obstructiva del sueño en población adulta chilena. Rev Chil Enferm Respir 2017; 33: 275-83.
6.- JORQUERA J. Síndrome de apnea obstructiva del sueño [Internet]. [citado el 4 de agosto de 2018]. Disponible en: http://publicacionesmedicina.uc.cl/Boletin/20072/SindromeApnea.pdf.

7.- MAYO CLINIC. Apnea del sueño - Síntomas y causas [Internet]. [citado el 4 de agosto de 2018]. Disponible en: https://www.mayoclinic.org/es-es/diseases-conditions/sleep-apnea/symptoms-causes/syc-20377631.

8.- MENON SM, SAMPANGIRAMAIAH S, MATHEW M. Cross Sectional Observational Study Performed to See for Relation of Mallampati Score and Extended Mallampati Score with Body Mass Index [Internet]. [citado el 3 de agosto de 2018]. Disponible en: https:// www.ncbi.nlm.nih.gov/pmc/articles/PMC5483783/.

9.- PEREIRA DC, DE ARAUJO MF, DE FREITAS RW, TEIXEIRA CR, ZANETTI ML, DAMASCENO MM. Neck circumference as a potential marker of metabolic syndrome among college students. Rev Lat Am Enfermagem 2014; 22:973-9.

10.- GOIC A, CHAMORRO G, REYES H. Semiología médica. $3^{\text {ra }}$ ed. Santiago: Mediterráneo, 2011; pp. 36982.

11.- FLEMONS WW, WHITELAW WA, BRANT R, REMMERS JE. Likelihood ratios for a sleep apnea clinical prediction rule. Am J Respir Crit Care Med 1994; 150: $1279-85$

12.- CHUNG F, ABDULlAH H, LIAO P. STOP-Bang Questionnaire: A practical Approach to Screen for Obstructive Sleep Apnea. Chest 2016; 149: 631-8

13.- RAMACHANDRAN SK, JOSEPHS LA. A metaanalysis of clinical screening tests for obstructive sleep apnea. Anesthesiology 2009; 110: 928-39.

14.- SALDIAS F, GASSMANN J, CANELO A, MONASTERIO J, DÍAZ O. Evaluación de los cuestionarios de sueño en la pesquisa de pacientes con síndrome de apneas obstructivas del sueño. Rev Med Chile 2018; 146: 1123-34.

15.- RUIZ A, HIDALGO P, PÁEZ S, PERILLA A, GONZÁLEZ C. Correlación de la escala de somnolencia de Epworth con el diagnóstico y severidad del síndrome de apnea hipopnea obstructiva del sueño (SAHOS). Revista Colombiana de Neumología 2013; 25: 10-15.

16.- NUCKTON TJ, GLIDDEN DV, BROWNER WS, CLAMAN DM. Physical examination: Mallampati score as an independent predictor of obstructive sleep apnea. Sleep 2006; 29: 903-8.

Correspondencia a:

Dr. César Maquilón O.

Servicio Médico Quirúrgico, Clínica Dávila.

Av. Recoleta 464. Comuna de Recoleta, Santiago, Chile. Código Postal: 8431657

Email:cmaquilon87@hotmail.com 